\title{
Control architecture of power systems: Modeling of purpose and function
}

\author{
Heussen, Kai; Saleem, Arshad; Lind, Morten
}

Published in:

IEEE Power \& Energy Society General Meeting, 2009.

Link to article, DOI:

10.1109/PES.2009.5275963

Publication date:

2009

Document Version

Publisher's PDF, also known as Version of record

Link back to DTU Orbit

Citation $(A P A)$ :

Heussen, K., Saleem, A., \& Lind, M. (2009). Control architecture of power systems: Modeling of purpose and function. In IEEE Power \& Energy Society General Meeting, 2009.: PES '09. (pp. 1-8). IEEE.

https://doi.org/10.1109/PES.2009.5275963

\section{General rights}

Copyright and moral rights for the publications made accessible in the public portal are retained by the authors and/or other copyright owners and it is a condition of accessing publications that users recognise and abide by the legal requirements associated with these rights.

- Users may download and print one copy of any publication from the public portal for the purpose of private study or research.

- You may not further distribute the material or use it for any profit-making activity or commercial gain

- You may freely distribute the URL identifying the publication in the public portal 


\title{
Control Architecture of Power Systems: Modeling of Purpose and Function
}

\author{
Kai Heussen, Student-Member, IEEE, Arshad Saleem, Student-Member, IEEE, and Morten Lind
}

\begin{abstract}
Many new technologies with novel control capabilities have been developed in the context of "smart grid" research. However, often it is not clear how these capabilities should best be integrated in the overall system operation. New operation paradigms change the traditional control architecture of power systems and it is necessary to identify requirements and functions. How does new control architecture fit with the old architecture? How can power system functions be specified independent of technology? What is the purpose of control in power systems? In this paper, a method suitable for semantically consistent modeling of control architecture is presented. The method, called Multilevel Flow Modeling (MFM), is applied to the case of system balancing. It was found that MFM is capable of capturing implicit control knowledge, which is otherwise difficult to formalize. The method has possible future applications in agent-based intelligent grids.
\end{abstract}

Index Terms-Functional Modeling, Requirement analysis, Modeling methods, Frequency Control, Smart Grid Concepts

\section{INTRODUCTION}

$\mathbf{T}$ HE transition of power systems today to the "smart" energy systems of the future has received much attention from industry, research and public institutions in recent years. The interest is a result of the need for replacement of old equipment on one side, and of new requirements associated with sustainability for future energy systems, on the other.

In this context, particularly in the US and Europe, many projects have been started that aim at developing new technologies and concepts to shape the idea of the "Smart Grid". US projects tend to emphasize on the development of new concepts and architectures ${ }^{1}$ for grid components, business interoperability as well as restructuring markets for more realtime operation. In comparison, the focus in the European Smart Grids platform ${ }^{2}$ is rather on the active integration of renewable energies (REN) ${ }^{3}$ and distributed resources (DR $)^{4}$ and to bring about an evolution of the existing system architecture.

In Denmark specifically, the political goal of 50\% share of wind energy by 2025 has inspired the ECOGRID project. This project, funded by the danish transmission system operator ${ }^{5}$, aims at preparing the danish power system for this challenge [1].

All authors are from the Department of Electrical Engineering and affiliated with the Centre for Electric Technology, Technical University of Denmark, 2800 Kgs. Lyngby, Denmark.

e-mail: $\{$ kh,asa,mli $\}$ @elektro.dtu.dk

${ }^{1}$ e.g. EPRI's Intelligrid http://intelligrid.epri.com/ or the GridWise Alliance (http://www.gridwise.org/)- particularly the associated Architecture Council (http://www.gridwiseac.org/)

${ }^{2} \mathrm{http}: / /$ www.smartgrids.eu/

${ }^{3}$ e.g. EWIS (http://www.wind-integration.eu/) and TradeWind (http://www. trade-wind.eu/)

${ }^{4}$ e.g. the projects FENIX (http://www.fenix-project.org/) or ADDRESS (http://www.addressfp7.org/).

${ }^{5}$ Energinet.dk
The recently ended Phase I of the ECOGRID project included a work package on "System Architecture". This work package was comprised of a review of "innovative technologies", a "requirement analysis", and an outlook on "possible solutions". It has been emphasized that there is a need for identifying the requirements to define the architecture of the future system [1], [2]. When discussing system architecture, enabling technologies should be known. It is crucial, however, to assess the technologies and to analyze the anticipated needs in order to redefine the overall goals and to specify the functions required of solutions. This specification of functional requirements must be clear, concise and generic to leave freedom for future design innovations, especially for the adoption of future sustainable technologies. Further, it was concluded that concepts, methods and tools are needed that enable design and evaluation of system architecture.

\section{A. Accommodating New Technology}

Major shifts in technology motivate system redesign. For instance, power electronics revolutionize the way energy flows can be controlled, both in power generation and transmission. Also, with the increased amount of REN and DR, a large number of technologies have been and are being developed that enable a controllable consumption and generation of energy in general (e.g. frequency responsive demand, demand clusters, vehicle to grid, etc.). Another class of new technologies regards the supervisory control of power systems on the larger scale [1], such as PMU measurements and online state estimation. Here, also control theory has brought potential for "smarter" power system automation, improving both stability and resource utilization [3]. Information and communication technology (ICT) can be regarded as an enabling technology for many of the new concepts listed here.

Many of these new technologies bring desirable capabilities [4], which are not naturally supported by the traditional power system and energy markets. And often they are of a scale too small to be recognized by energy markets or to be controlled by grid operators.

\section{B. Challenges for Control Architecture}

A major issue for system integration is manageability or controllability of these technologies in the context of an already complex power system. The active integration of these additional resources requires new concepts for control and supervision.

In recent years many new concepts have been developed that aim at tackling this challenge. Most of these concepts can be categorized as aggregation approaches of two kinds: 
(1) Aggregation based on the physical location of resources (in the grid) ${ }^{6}$, and (2) commercial aggregation concepts rather based on the generation patterns and capabilities resources [5], [6] $]^{7}$. The former are aimed at improving the technical operation of the system, and research in this area is of rather technical nature. Whereas, the latter are striving for a profitable participation in energy markets, such that research in this direction focuses on the economical and market-operation principles.

It is generally difficult to evaluate and integrate such complex technologies, particularly when originating from different backgrounds. In order to do that one needs to understand purposes and functions these systems.

In this paper, we present a framework and modeling approach for describing the relations between purpose and functions. A particular strength of the modeling tool used here, called Multilevel Flow Modeling (MFM), is that it provides a meaningful representation of control functions.

By applying this functional modeling approach to the frequency control mechanism, as described in the literature, we show how the network of control objectives and functions composes the system to function as one unit. The modeling technique can be a bridge from values to design as it makes possible to explicate the relation between purposes and functions of the technical system.

In Section II the modeling method is introduced and explained. The rest of the paper is devoted to illustrating the application of functional modeling to power systems. In Section III-A we analyze power system goals on the highest level, in order to gain a clear formulation of the "ends" of electrical energy systems. Next, as the main contribution, a MFM model of frequency control is developed in Section III-B. Finally the presented results and are discussed and future work is motivated in Section IV.

\section{Multilevel Flow Modeling}

Multilevel Flow Modeling (MFM) is an approach to modeling goals and functions of complex industrial processes involving interactions between flows of mass, energy and information [7]-[12]. MFM has been developed to support functional modeling [13] of complex dynamic processes and combines means-end analysis with whole-part decompositions to describe the functions of the process under study and to enable modeling at different levels of abstraction. Process functions are represented by elementary flow functions interconnected to form flow structures representing a particular goal oriented view of the system (Figure 1a)). Flow structures are interconnected in a multilevel representation through meansend relations, causal roles and control functions and structures (Figure 1b)). MFM is founded on fundamental concepts of action [11] and each of the elementary flow and control functions can be seen as instances of more generic action types. The views represented by the flow structures, functions, objectives and their interrelations comprise together a comprehensive

\footnotetext{
${ }^{6}$ i.e. MicroGrids, Cells, Technical Virtual Power Plants, ...

${ }^{7}$ e.g. (Commercial) Virtual Power Plants
}

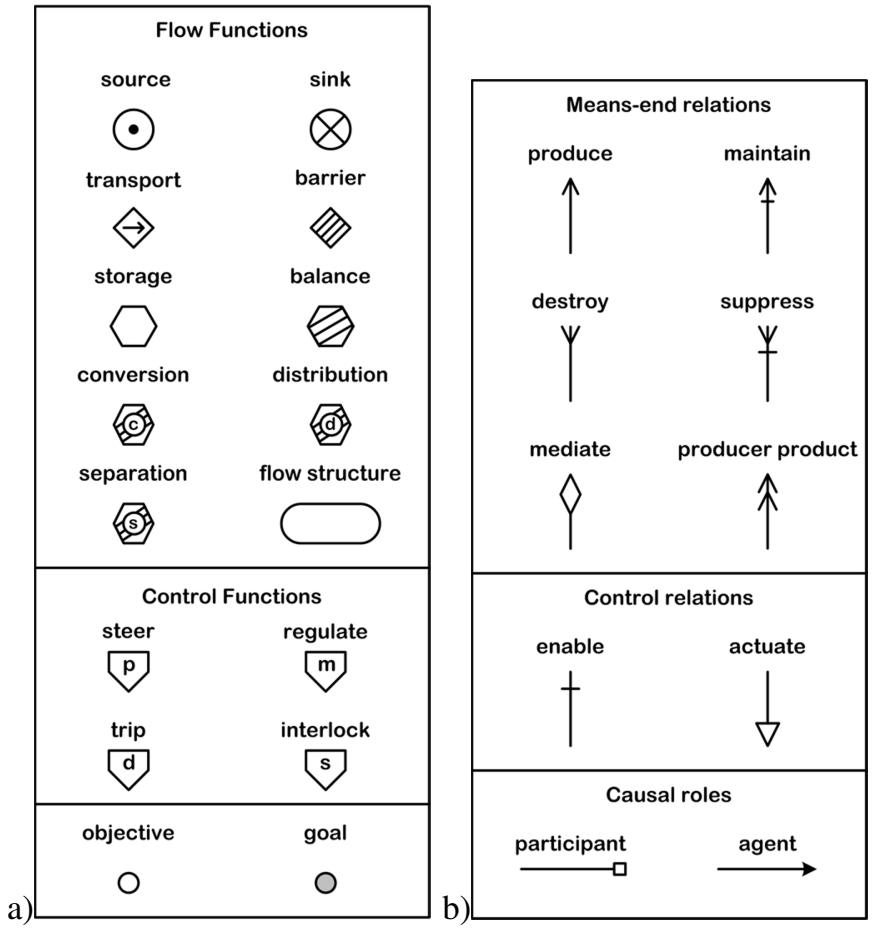

Fig. 1. a) MFM entities and b)MFM relations

model of the functional organization of the system represented as a hypergraph. It should be noted that MFM is a formalized conceptual model of the system which supports qualitative reasoning about control situations [14], [15].

MFM has been used to represent a variety of complex dynamic processes including fossil and nuclear power generation [16]-[18], oil refineries [19], chemical engineering [15], [20] and biochemical processes [21].

Application of MFM includes model based situation assessment and decision support for control room operators [22], hazop analysis [23], alarm design [24] and alarm filtering [25] and planning of control actions [16], [26]. MFM is supported by knowledge based tools for model building and reasoning [12].

MFM has been applied in power systems by Larsson [27] without explicit representation of control functions. Here we show that the capability of representing control is essential for capturing the functional complexity of power systems.

Application of MFM in power systems is envisioned to further intelligent agent solutions in power systems control. MFM models could support situation-awareness of agents, for example to enable reasoning about appropriate responses in fault situations.

\section{A. Demonstrating MFM principles by a small example}

Application of the MFM concepts is illustrated in the following by a simple example in Figure 2 below. The model represents the objectives and functions of a water circulation loop in a heat transfer system. It is assumed that the water is circulated by an oil lubricated pump. The example illustrate how the MFM model provides a comprehensive understanding of the purpose and functions of the circulation loop and its 
subsystems. On an overall level the model can be seen as composed of three sub-models representing different views on the water circulation system.

The first view (starting from the top) represents systems aspects related to water circulation and comprises the flow structure labeled MFS1, the produce relation and the objective O1. This part of the models represents the overall objective of the water circulation, which is to produce a flow of water. The flow structure contains the functions provided to circulate the water. In this simplified model the transport function T1 is the means used for water circulation.

The second view is partially overlapping with the first view because what is seen here as a means (the transport T1) is in the second view seen as an end. Transport T1 is related to the means of transport which is the pumping represented by the energy flow structure EFS1). T1 and EFS1 is therefore related by a type of means-end relation called a producer-product relation in MFM. The flow structure EFS1 is decomposed into the flow functions representing the services provided by components of the pump system (including the energy supply) in order to achieve the end, the transportation of water represented by $\mathrm{T} 1$.

The third view is related with the second view through an enabling relation and an associated objective $\mathrm{O} 2$ which is the end to be achieved by the functions contained in the flow structure MFS2. The flow structure MFS2 represents the functions involved in the lubrication of the pump and the objective $\mathrm{O} 2$ represents the condition that should be fulfilled in order to ensure that the pump is properly lubricated. A condition which should be satisfied in order to enable the pump to provide its functions. The flow functions inside MFS2 accordingly represents the functions of the pump lubrication system.

Even though the example does not utilize all the concepts of MFM, it demonstrates the power of MFM to represent in a clear and logical way knowledge about the goals and functions of a system. The MFM modeling language has a strong syntax which define rules for combining the different entities and relations of the language into a consistent model.

\section{B. Control Functions}

The modeling example above described the functions of the components and subsystem which contributed to the overall objective of the system (deliver water flow). No consideration was accordingly given to the purpose and function of control systems in meeting this objective. As is well known control systems are important for ensuring that process objectives are met in spite of uncertainty and disturbances in the process. This is actually the basic reason for using control systems. MFM has a set of functions which can be used to represent control system functions. We will use the example above to illustrate how some these concepts are used.

Assume that we need to keep the lubrication flow in the pump within specified limits in order to avoid pump problems. An engineering solution to this problem could be to use a regulator measuring the oil flow and controlling the speed of the oil pump. The function of the regulator is to maintain oil

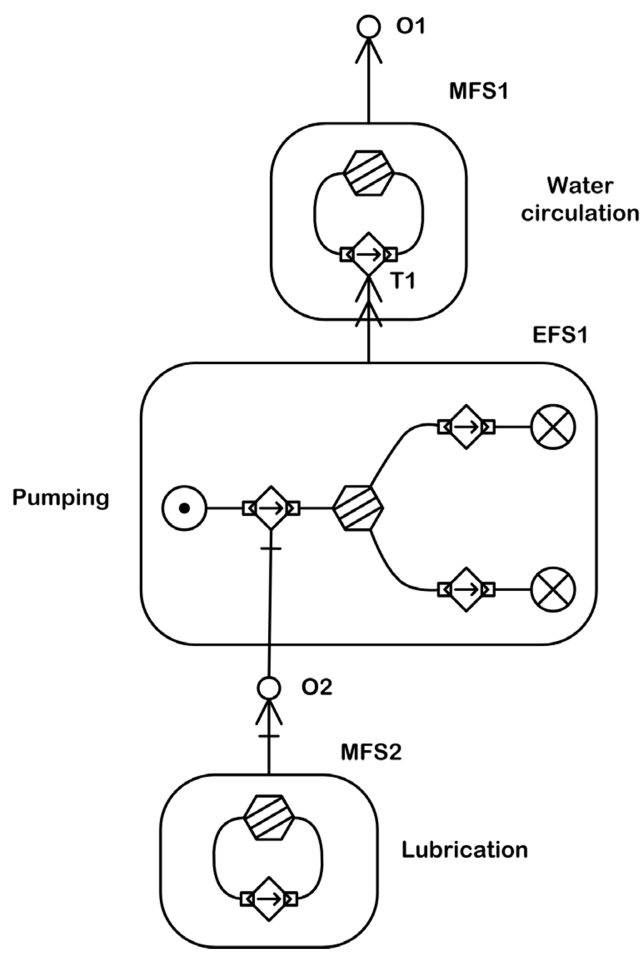

Fig. 2. MFM model of a water circulation loop

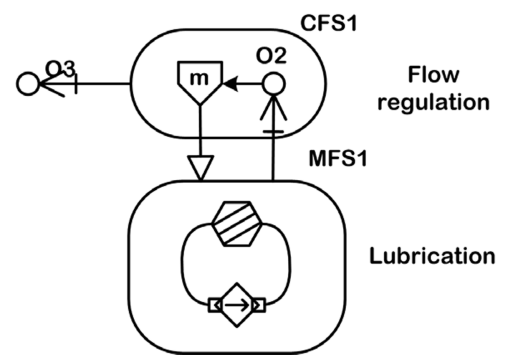

Fig. 3. MFM model of the regulated lubrication system

flow within limits. This function can be modelled in MFM as shown in Figure 3.

Note that we have introduced a new objective $\mathrm{O} 3$ in addition to the original objective $\mathrm{O} 2$. It is very important to emphasize the fundamental difference between these two objectives. O2 is "process" objective specifying the value range within the lubrication flow should be kept. In contrast O3 in a "control" objective specifying the performance required of the regulated process. The control objective could specify stability margins etc. and other control attributes specifying the desired performance of the regulator (see also Lind [9]).

It should be stressed that the "loop" formed by the maintain and the actuate relations connecting the mass flow and the control flow structures are conceptual relations and is therefore not a representation of the function or structure of a feedback loop. The concept of feedback is connected with signal or information flow. Control functions shown here do not describe information flow but the purpose of the control action (to regulate). 


\section{Purpose And Functions of Power Systems}

In the following we will demonstrate, how MFM can be applied to power systems. In order to refer to a rather generic power system the modeling was based on the descriptions derived from reference [28].

The process of modeling in MFM is an iterative process, it can be started in principle at any level of means-ends decomposition. An outcome of the modeling is a clear understanding of functions at various levels of abstraction.

The results of the analysis are presented in two stages: First, high-level system objectives are discussed, and then the it will be shown how MFM can be used to model the frequency control hierarchy.

\section{A. Objectives of an Electrical Power System}

Usually the location of energy sources is distant from where energy is needed. Electricity is a natural choice for energy delivery, because it can be transported effectively and it can be converted from and to mechanical energy with high efficiency ${ }^{8}$.

The purpose of electrical energy systems is thus the timely provision of electrical energy to satisfy the demand for different forms of energy. The function of the electrical energy system describes how the system serves its purpose. That is,

the function of an electric power system is to convert energy from one of the naturally available forms to the electrical form and to transport it to the points consumption. [28]

Kundur further elaborates that the power system should meet "fundamental requirements" as follows (p.9, [28])

1) ... meet the continually changing load demand of active and reactive power ... [while considering that, (edt.)] electricity cannot be stored conveniently in sufficient quantities. [...]

2) ... supply energy at minimum costs and minimum ecological impact

3) The "quality" of power must meet minimum standards with regard to $[. .$.

\section{(a) constancy of frequency}

(b) constancy of voltage;

(c) level of reliability

The scope of these requirements encompasses different time ranges and scopes of planning and comprises technical, economical and societal (ecological) goals.

Technical objectives tend to dominate the operational requirements, whereas economical objectives tend to be oriented more on scheduling and planning. Ecology considers the whole life cycle, but it is not always straightforward how this requirement is to be interpreted in practice. Let us therefore further differentiate objectives by: operation, scheduling, planning and system design.

The categorization of requirements and goals into "economical" and "technical" can actually be derived from different

\footnotetext{
${ }^{8}$ The transformation of thermal or chemical energy is not as efficient. District heating systems are a good counter-example, that illustrates that electricity is not always the most efficient form of energy distribution.
}

values that are associated with these goals [29]. In abstraction from economical, technical and societal categories the authors identified the following values in the context of energy systems:

1) Security of energy supply;

2) Overall resource efficiency of the energy system; and

3) Sustainability of system structure, operation and planning.

These values express the most fundamental sources of "requirements" we could derive, and they are technology independent. The suggested prioritization was observed for instance by how these values have been considered historically in the electrical power systems context ${ }^{9}$.

Let us elaborate a bit on the interpretation of these three values:

1. Security (availability) of energy supply relates to the basic human value of security, the security that energy is available when needed. In a more long term perspective, it also means security of access to energy resources, for example.

2. Resource efficiency relates to the general understanding that resources are limited and that efficient utilization frees resources for other purposes. Resources could be natural (e.g. energy or material), but could also be human or monetary resources. A typical means of evaluating system efficiency is the creation of institutions or market instruments to enable means of monetary resource allocation and evaluation. Economical evaluation is however limited to the extent in which costs and benefits can reasonably be quantified.

3. The concept of sustainability is rather new in the context of power systems, but it has a long tradition in the provision of energy resources. It is important to include objectives of this kind to give space for reasoning about appropriateness of technologies and the application of methodologies that go beyond the capacity of econometric tools.

Criteria formulated in terms of values are pervasive in principle. That means, they affect all system objectives, functions and realization independently.

Now, given these value-criteria and categories, how do we interpret the "fundamental requirements" quoted above?

1) "Meeting the continually changing demand" clearly is an operational objective and it relates to security of energy supply. We take this as the central goal of a power system:

$\mathrm{g}_{1}$ : Supply electrical energy as demanded.

2) The requirements regarding "costs" and "ecology" are high-level criteria and are basically equivalent to the value statements on resource efficiency and sustainability, respectively.

3) The requirements relating to the "quality of power", are rather mixed. Quality requirements (a) and (b), constancy of voltage and frequency, respectively, are strictly functional requirements. Point (c) "reliability", however, can be interpreted in many ways:

- If subordinated to power quality it is a functional requirement.

${ }^{9}$ It may not be a "natural" prioritization, but it is unclear if a such a "natural" prioritization exists after all. 
- It can also be seen as a high-level objective, derived from security of supply / availability.

- Some aspects of reliability could characterize the specification of control objectives, such as performance, or stability, which includes those objectives related to stabilizing the network as a whole. These objectives which would be subordinated to $\mathrm{g}_{1}$ as a purpose.

The following modeling focuses on achieving an operational understanding of objective $\mathbf{g}_{\mathbf{1}}$.

\section{B. Control Functions for Balancing Generation and Demand}

Following the discussion above, we now start developing a functional model of the control structure of electrical energy systems. The focus is on the frequency control mechanism, which is directly related to the high-level goal of supplying as much energy as demanded. To put this model in context, we shall first analyze common representations of these control structures from the literature as given in [28].

A common and detailed illustration of the power system control functions is given in Figure 4. It shows a composition of several subsystems (boxes) interconnected by signals (arrows). It may be interpreted as follows: On the top of the diagram we find the "System Generation Control" which receives a set of input signals and issues "supplementary control" signals to as inputs to generating units. One of the input signals is called "generation schedules", which should represent the operating points of all generators participating in the system control. The other inputs comprise information on the system operating state, received from the "Transmission Controls". The central part of the diagram shows subsystems of a power plant (Generation unit) considered relevant for power system control. This includes the prime mover as source of energy and generating torque and the associated generation control system, which receives the rotor speed and supplementary control as control inputs. The generator, receiving this torque from the prime mover (shaft power), feeds back the rotor speed. The generator further receives inputs and feeds back to its excitations system and controls, and finally, it emits an electrical power and voltage as outputs of the power plant subsystem.

Further, the "transmission controls" receive this electrical power as input information for their control responsibilities, which includes the control of voltage and reactive power. This simplified view suggests a subordinated role of the transmission controls, for example, omitting the role of the generation units in voltage control. In this paper we also limit the scope of modeling to the active power / energy related system functions. That means the subsystems and signals marked with thin dash-dotted lines are only included implicitly in the following.

The model in Figure 4 is based on the signal-flow type of diagram, where the arrows present signals and the boxes represent systems which generate or transform signals. This type of diagram origins from signal processing and is often used to explain the composition of control systems. The naming of the boxes and signals ascribes meaning to them, and

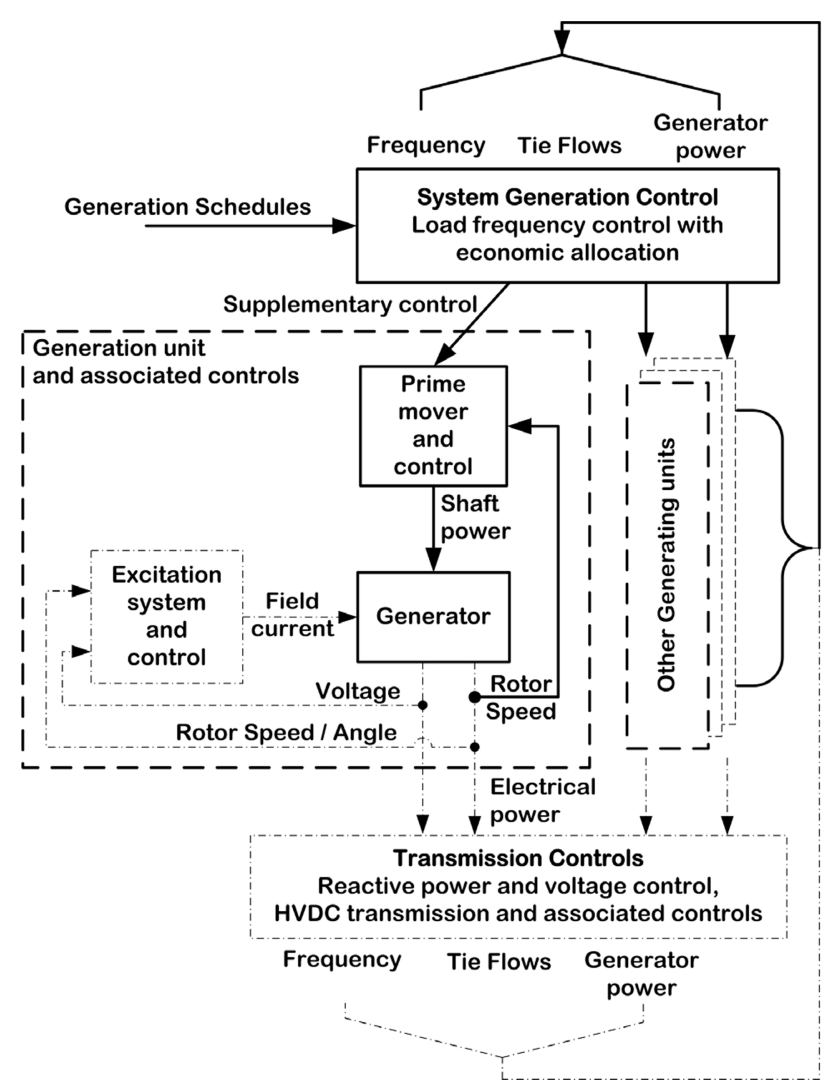

Fig. 4. Subsystems of a power system and associated controls (adapted from [28], Fig.1.2). The subsystems shown with dash-dotted lines are not modeled explicitly in this paper.

their relation with each other can be interpreted as commandchain or physical interconnection. This kind of interpretation of Figure 4 was given above.

However, the functions represented in this type of diagram can formally only be interpreted as signal processing functions. One could argue that it is often possible to interpret the intentions implemented in the design of a control system from a signal-flow diagram. In this case, the intentions are then inferred from conceptual schemes of control engineering. Yet, the intentionality is only implicit in the ordering of signal flow structures. In fact this type ordering is prone to misinterpretation, for example when a system redesign is attempted without considering the underlying design objectives [11].

Signal-flows are also used to suggest control hierarchies and control roles in the modeled system. Figure 5 illustrates the hierarchical structure of power system control by a flow of command signal flows and a command hierarchy in an organigramme. This control hierarchy can be divided systematically into control levels, depending on level of abstraction, the relevant time scales and type of control tasks performed [3], [30], [31]. This approach is meaningful for complex automation systems and it can also be found in other industrial automation systems [32].

1) Functional Structure of the Energy System: In contrast to the types of diagrams used above, functions and purposes of systems and subsystems are modeled explicitly in functional 


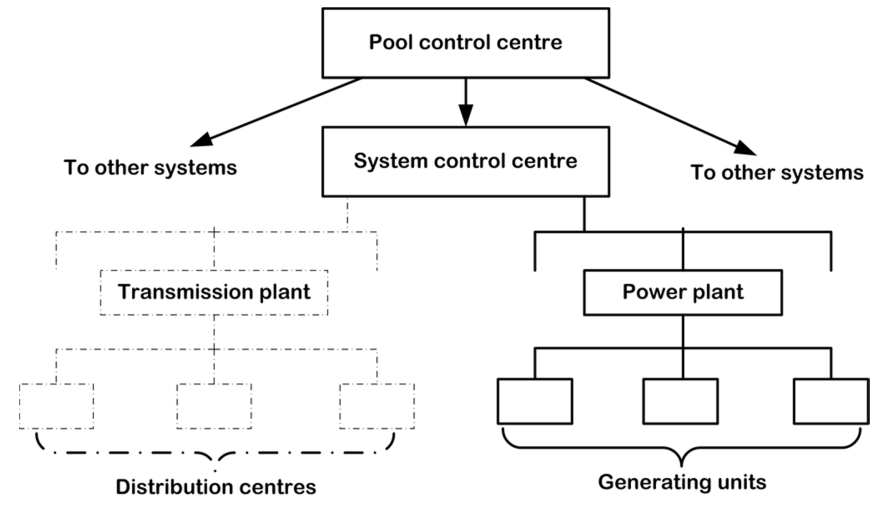

Fig. 5. A representation of control hierarchy in power systems from the literature (adapted from [28], Fig. 1.4).

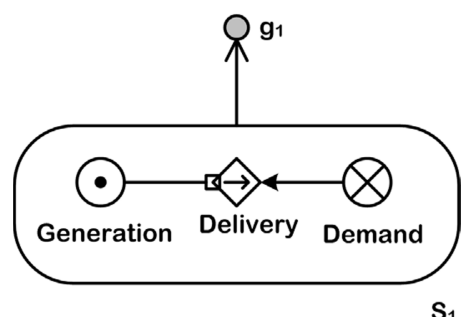

$\mathrm{S}_{1}$

Fig. 6. High-level view of the energy system (MFM model).

models. Multi-level Flow Modeling (MFM) provides rich semantics to model the relations of utility between systems and subsystems. The means-ends decomposition is possible both in terms of intention, as goal-oriented action, and in terms of intentional composition of physical functions in energy and mass flow functions.

The most high-level view of the multilevel flow model is shown in Figure 6 . The energy system is here described by an energy flow structure $\mathbf{S}_{1}$, describing the process view, and its association with goal $\mathbf{g}_{1}$ : Satisfy energy demand, employing the means-ends relation: produce. $\mathbf{S}_{\mathbf{1}}$ comprises three energy flow functions: A source (Generation), a transport function (Delivery), and a sink (Demand). The flow functions are interconnected by causal relations: Generation is a participant, supplying energy to the transport function, whereas Demand is an agent causing the energy flow. These causal roles imply that generation is supposed to be following the load demand. This causal role is realized by the frequency control functions that will be analyzed below. The transport function in $\mathbf{S}_{\mathbf{1}}$ represents the action of power-delivery at any time.

2) Abstract Model of Frequency Control: The flow structure and goal introduced above represent the overall function of the electrical energy system. This function is of course dependent on mechanisms that bring about the intended causality, to satisfy the goal. That mechanism is frequency control.

The purpose of frequency control is accordingly represented by the causal relations between generation and demand in the flow structure $\mathbf{S}_{\mathbf{1}}$ in Figure 6 . This purpose is achieved by a cascade from centralized to decentralized control and coordination functions. The decentralized, low-level, control functions are implemented on the generators and are known

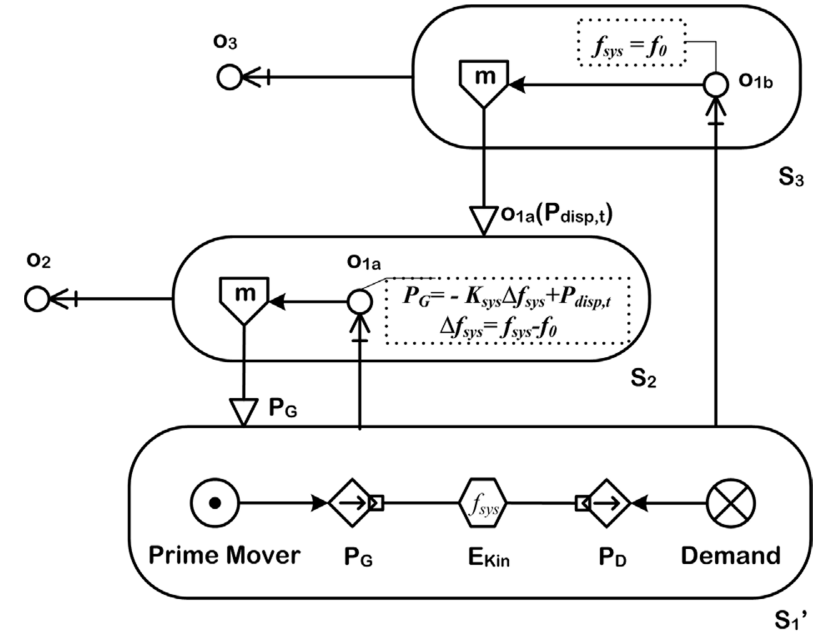

Fig. 7. Abstract MFM model of the system balancing hierarchy.

as frequency droop control or primary frequency control. The more central control functions are associated with secondary frequency control, inter-area balancing, economical allocation et cetera. Control functions on this level have been generalized as "corrective control" in [3]; in the following we will refer to it as system balancing. The coordination of these two control functions is possible due to the kinetic energy stored $\left(\mathbf{E}_{\mathbf{k i n}}\right)$ in the generators of the power system and the associated synchronous $^{10}$ frequency $f_{\text {sys }}$.

An MFM model of this composition is shown in Figure 7. Here, the flow structure $\mathbf{S}_{1}{ }^{\prime}$ shows an expansion of the flow structure $\mathbf{S}_{1}$ in Figure 6 , where the energy source (Generation) has been expanded. The frequency droop control is represented by the control flow structure $\mathbf{S}_{\mathbf{2}}$ and system balancing is modeled as control structure $\mathbf{S}_{\mathbf{3}}$. The objectives associated with $\mathbf{S}_{\mathbf{1}}{ }^{\prime}, \mathbf{o}_{\mathbf{1}} \mathbf{a}$ and $\mathbf{o}_{\mathbf{1 b}} \mathbf{b}$, are a decomposition of the above stated purpose of frequency control. This purpose can be formalized as follows:

$$
\mathbf{o}_{1}: \quad P_{G} \stackrel{!}{=} P_{D}
$$

where $P_{D}$ is the power consumed by the demand, and $P_{G}$ is the shaft power of the generators. This equation is a statement of intention, which is expressed by the exclamation mark $(\stackrel{!}{=})$.

The separation between frequency droop control and system balancing is based on a decomposition of (1):

$$
P_{G}=-K_{\text {sys }} \Delta f_{\text {sys }}+P_{\text {disp }, t},
$$

with $\Delta f_{\text {sys }}=f_{\text {sys }}-f_{0}$ is the frequency deviation, $K_{\text {sys }}=$ $\frac{1}{R_{s y s}}$ is the system droop constant and $P_{d i s p, t}$ is the total power dispatch by the system balancing function. This decomposition leads to the objectives $\mathbf{o}_{1 \mathbf{a}}$ and $\mathbf{o}_{1 \mathbf{a}}$ of droop control and system balancing, respectively.

Droop control or primary frequency control is necessary for the mitigation of larger short-term deviations in the balance between load and demand. The response is coordinated by an adequate stetting of the droop constants, such that a required system droop constant $R_{s y s}=\frac{1}{K_{s y s}}$ is achieved

\footnotetext{
${ }^{10}$ This synchronous operation is a load-sharing mechanism, realized by lower-level functions.
} 
(Section III-B3). The objective is thus to achieve the droop characteristic:

$$
\mathbf{o}_{1 \mathbf{a}}: \Delta f_{\text {sys }} \stackrel{!}{=} P_{G}=R_{\text {sys }} \cdot\left(P_{\text {disp }, t}-P_{D}\right),
$$

The primary frequency-control $\left(\mathbf{S}_{\mathbf{2}}\left(\mathbf{o}_{1 \mathbf{a}}\right), \mathbf{o}_{2}\right)$ ensures that the frequency deviation matches the droop setting and power dispatch. It does so by means of adjusting the prime mover $\mathbf{P}_{\mathrm{G}}$, the shaft power input to the generators, using control according to the performance specified in $\mathbf{o}_{2}$. As a result, the frequency reflects the mismatch between demand and dispatched power. The power dispatch is to be adjusted by the system balancing $\mathbf{S}_{\mathbf{3}}$.

Following (2), the objective $\mathbf{o}_{1}$, i.e. matching dispatched generation with demand, is equivalent to returning the frequency to its nominal value:

$$
\mathbf{o}_{\mathbf{1 b}}: f_{\text {sys }} \stackrel{!}{=} f_{0},
$$

Thus, system balancing is aimed at bringing the frequency back to its nominal value by means of adjusting the power dispatch. The performance objective $\mathbf{O}_{3}$ specifies how the control structure $\mathbf{S}_{\mathbf{3}}$ should achieve the control objective $\mathbf{o}_{\mathbf{1 b}}$, which could be, for example, a formulation of the time-scales associated with primary, secondary and tertiary frequency control, or economic allocation criteria.

3) De-aggregation to Represent Individual Units: Above, all generators were aggregated into one. In this section we show the system view of frequency control for an individual generator. The aggregation of the previous section is split into two sources and two transport functions: G1, $P_{G 1}$ and $P_{G r e s t .}$ The inertia (energy storage) remains aggregated in this view (Figure 8).

For this case, equation (2) can be decomposed into

$$
P_{G}=-\left(\sum_{i=1}^{N} K_{i}\right) \Delta f_{\text {sys }}+\sum_{i=1}^{N} P_{d i s p, i} .
$$

We have therefore two system constants that can be coordinated independently on the higher aggregation levels:

$$
\text { 1) } \frac{1}{R_{\text {sys }}}=K_{\text {sys }}=\sum_{i=1}^{N} K_{i} \quad \text { 2) } P_{d i s p, t}=\sum_{i=1}^{N} P_{d i s p, i} \text {. }
$$

The coordinated droop of all synchronous generators is the sum of the individual responses. The balancing control $\mathbf{S}_{\mathbf{3}}$ actuates the generators independently of their contribution to primary control. The frequency gets restored by balancing control, as a result all primary controllers get back into balance.

\section{Discussion AND CONCLUSION}

The analysis of power systems presented here presents a new angle on control design starting with the question: What is purpose of power systems? This seemingly remote analysis of values revealed two important facts: (1) there is a hierarchy among the typically believed standard objectives of power system operation; and (2) whenever new power system (control) objectives are defined, a choice based on values is made. This rigorous ends-means approach set an anchor for the

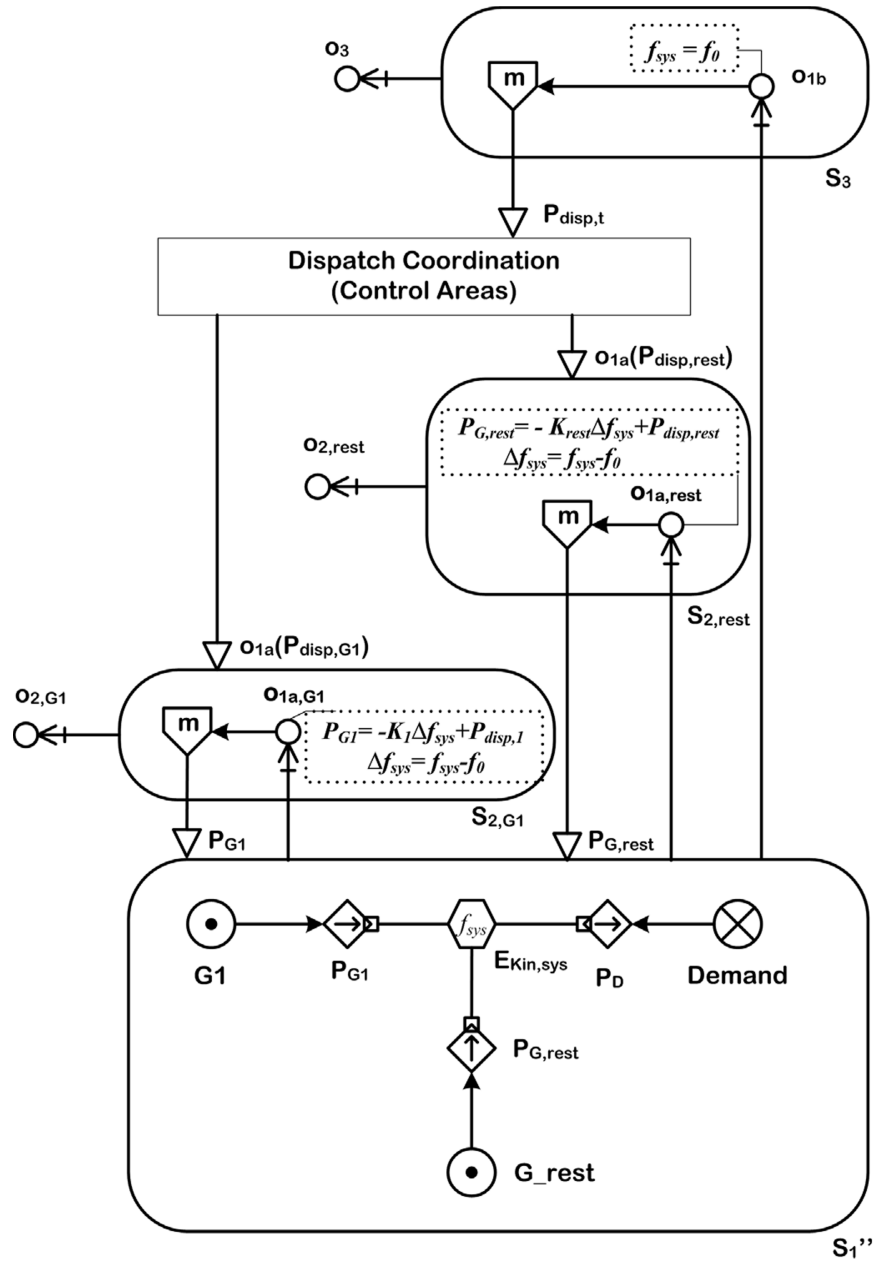

Fig. 8. Distributed frequency control. The generator control structures $\mathbf{S}_{2, \mathbf{G 1}}, \mathbf{S}_{\mathbf{2}, \mathbf{G r e s t}}$ locally adjust their generation according to their respective power setpoint and local droop setting, based on the common system frequency.

analysis using MFM. The following analysis of the frequency control clarifies the concepts of frequency control. Seen in the larger picture, this model could contribute with categories of control functions for new active power control technologies (for example for of Wind Turbines).

So far, with frequency control, only a model of one of the simplest control functions in the domain has been presented. Some of the further modeling challenges addressed in future work are:

- Load-angle stability: a deeper analysis of control functions that enable synchronous operation.

- Reactive power and voltage control: this modeling task comprises two challenges: (1) a MFM model of reactive energy flows needs to be developed that is consistent with the common understanding of reactive power; and (2) a model of the spatially distributed control of voltage.

- Even though the balancing functions described here are in line with the description derived from [28], the complex coordination patterns of inter-area balancing and program responsibility require a more detailed modeling of the control structures.

This is the first study of control functions in power systems 
using MFM. The study is part an ongoing work and will be expanded to more control functions in order to obtain a comprehensive understanding of control architecture in power systems. We conclude that MFM can be an effective analytical tool in the development and evaluation of new technologies for existing and future power systems.

\section{REFERENCES}

[1] Z. Xu, M. Gordon, M. Lind, and J. stergaard, "Towards a danish power system with $50 \%$ wind - smart grids activities in denmark," in Proceedings of the IEEE PES General Meeting 2009, 2009, (invited panel paper).

[2] M. Lind, T. Ackermann, P. Bach, H. W. Bindner, Y. Chen, R. GarciaValle, M. Gordon, K. Heussen, P. Nyeng, A. Saleem, P. E. Srensen, M. Togeby, I. Vlachogiannis, S. You, and Z. Xu, "Ecogrid.dk phase i wp2 report - system architecture," Energinet.dk, Tech. Rep., 2008.

[3] M. D. Ilic, "From hierarchical to open access electric power systems." Proceedings of the IEEE, Special Issue on Modeling, Identification, and Control of Large-Scale Dynamical Systems", vol. Volume 95, Issue 5, p. 1060 1084, May 2007.

[4] M. Braun, "Technological control capabilities of der to provide future ancillary services," Distr. Energy Resources, Int. Journ. of, vol. 3, pp. 191-206, 2007.

[5] D. Pudjianto, C. Ramsay, and G. Strbac, "Virtual power plant and system integration of distributed energy resources," IET Renew. Power Gener. vol. 1, no. 1, pp. 10-16, 2007.

[6] M. Braun and P. Strauss, "A review on aggregation approaches of controllable distributed energy units in electrical power systems," Distr. Energy Resources, Int. Journ. of, vol. 4, no. 4, pp. 297-319, 2008.

[7] M. Lind, "The use of flow models for design of plant operating procedures," in Proc. IWG/NPPCI Specialist meeting on procedures and systems for assisting an operator in normal and anomalous nuclear power plant operation situations, Garching, Federal Republic of Germany, December 1979.

[8] — , "The use of flow models for automated plant diagnosis," in Human Detection and Diagnosis of System Failures, J. Rasmussen and W. B. Rouse, Eds. Plenum Press, New York, 1981.

[9] - "Modeling goals and functions of complex industrial plant," Applied Artificial Intelligence, vol. 8, no. 2, pp. 259-283, 1994.

[10] the Institute of Measurement and Control, vol. 21, no. 4-5, pp. 171-180, 1999.

[11] — "Modeling goals and functions of control and safety systems in MFM," in Proceedings International Workshop on Functional Modeling of EngineeringSystems, Kyoto, Japan, January 25 2005, pp. 1-7.

[12] _ "Perspectives on multilevel flow modeling," in Proc. 4.th International Symposium on Cognitive System Engineering Approach to Power Plant Control (CSEPC2008), Harbin, Heilongjiang China, September 8-10 2008.

[13] _ "The what, why and how of functional modelling," in Proceedings of International Symposium on Symbiotic Nuclear Power Systems for the 21 'st Century (ISSNP), Tsuruga, Japan, July 9-11 2007, pp. 174-179.

[14] _ "Status and challenges of intelligent plant control," Annual Review of Control, vol. 20, pp. 23-41, 1996.

[15] A. Saleem, T. Us, and M. Lind, "Means-end based functional modeling for intelligent control: Modeling and experiments with an industrial heat pump," in Proc. IASTED conference on Intelligent Control Systems (ICS2007), Cambridge, Massachussets, USA, Nivember 21-23 2007.

[16] M. N. Larsen, "Deriving action sequences for start-up using multilevel flow models," Ph.D. dissertation, Department of Automation, Technical University of Denmark, 1993.

[17] J. Ouyang, M. Yang, H. Yoshikawa, Y. Zhou, and J. Liu, "Alarm Analysis and Supervisory Control Plan of PWR Plant," in Proceedings of CSEPC 2004, Cognitive Systems Engineering in Process Control, Sendai, Japan, Nocember 4-5 2004, pp. 61-68.

[18] J. Liu, H. Yoshikawa, and Y. Zhou, "Application of multilevel flow modeling to describe complex processes in a nuclear fuel cycle," in Proceedings CSEPC 2004 Cognitive Systems Engineering in Process Control, Sendai, Japan, November 4-5 2004, pp. 114-120.

[19] A. Gofuku and Y. Tanaka, "Application of Derivation Technique of Possible Counter Actions to an Oil Refinery Plant," in Proc. 4'th IJCAI Workshop on Engineering Problems for Qualitative Reasoning, Stockholm, 1999, pp. 77-83.
[20] L. W. Petersen, "Multilevel flow model of heat integrated distillation plant," MSc Thesis, Ørsted DTU, Automation, 2005.

[21] K. V. Gernaey, M. Lind, and S. B. Jørgensen, "Towards understanding the role and function of regulatory networks in microorganisms," in Computer Aided Process \& Product Engineering, L. Puigjaner and G. Heyen, Eds. Weinheim, Germany: Wiley-VCH, 2004.

[22] J. Petersen, "Situation assessment of complex dynamic systems using MFM," in Proceedings of 8th. IFAC/IFIP/IFPRS/IEA Symposium on Analysis, Design and Evaluation of Human-Machine Systems, Kassel, Germany, September 18-20 2001, pp. 645-650.

[23] N. L. Rossing, M.Lind, N. Jensen, and S. B. Jrgensen, "A goal based methodology for hazop analysis," in Proc. 4.th International Symposium on Cognitive System Engineering Approach to Power Plant Control (CSEPC2008), Harbin, Heilongjiang, China, September 8-10 2008.

[24] T. Us, N. Jensen, M. Lind, and S. B. Jrgensen, "Fundamental principles of alarm design," in Proc. 4.th International Symposium on Cognitive System Engineering Approach to Power Plant Control (CSEPC2008), Harbin, Heilongjiang China, September 8-10 2008.

[25] J. E. Larsson, "Diagnosis based on explicit means-end models." Artificial Intelligence, vol. 80(1), pp. 29-93, 1996.

[26] A. Gofuku and Y. Tanaka, "Development of an Operator Advisory System: Finding Possible Counter Actions in Anomalous Situations," in Proc. 5'th International Workshop on Functional Modeling of Complex Technical Systems, Paris, France, July 1-3 1997, pp. 87-97.

[27] J. E. Larsson, B. hman, and A. Calzada, "Real-time root cause analysis for power grids," in SECURITY AND RELIABILITY OF ELECTRIC POWER SYSTEMS, CIGR Regional Meeting, 2007.

[28] P. Kundur, Power System Stability and Control, EPRI, Ed. McGrawHill, Inc., 1994.

[29] N. Rescher, Introduction to Value Theory. Prentice-Hall Englewood Cliffs, NJ, 1969.

[30] F. C. Schweppe, "Power systems '2000': Hierarchical control strategies," IEEE Spectrum, vol. 6, pp. 42-47, 1978.

[31] B. Fardanesh, "Future trends in power system control," Computer Applications in Power, IEEE Journal on, vol. 02, pp. 24-31, 2002.

[32] D. Popovic and V. Bhatkar, Distributed Computer Control for Industrial Automation. M. Dekker, Inc., 1990.

Kai Heussen (M'07) is currently a Ph.D. student in Automation and Electric Energy Systems at the Department of Electrical Engineering at the Technical University of Denmark. He studied control engineering in Stuttgart and Toronto and received his degree of Engineering Cybernetics from the University of Stuttgart in 2007. His research interests include control theory, distributed control architecture and energy sytems with very high shares of renewable energy.

Arshad Saleem (M'07) is currently pursuing a Ph.D. degree from the Department of Electrical Engineering of the Technical University of Denmark. $\mathrm{He}$ received his bachelors degree in computer science from University of the Punjab Lahore, Pakistan and an M.Sc degree in Artificial Intelligence from Blekinge Institute of Technology Karlskrona, Sweden in 2003 and 2007 respectively. His research interests are intelligent systems applications to power system, power system analysis and distributed control.

Morten Lind is Professor of Automation at Department of Electrical Engineering at Technical University of Denmark and is associated Centre for Electric Technology. His research interests include automation design, supervisory control of complex industrial systems and infrastructures, and application of agent technology and knowledge based system in automation. 